\title{
Guideline Recommendations for Empirical Antimicrobial Therapy: An Appraisal of Research Evidence for Clinical Decision-Making in Ethiopia
}

\author{
Balew Arega · Asnake Agunie - Abraham Minda · Amdemeskel Mersha • \\ Alazar Sitotaw - Getachew Weldeyohhans - Ayele Teshome • \\ Kelemework Adane · Getachew Mengistu
}

Received: May 1, 2020 / Published online: June 22, 2020

(C) The Author(s) 2020

\section{ABSTRACT}

Introduction: The rapid spread of drug resistance is forcing standard treatment guidelines (STGs) to become more appropriate with due consideration of the evidence on the antimicrobial resistance (AMR) situation in Ethiopia. Therefore, we aimed to assess the local AMR recommendations in the STGs for empirical antibacterial prescriptions for the five common infectious syndromes. We also determined the quality of AMR reviews conducted in the country.

Methods: We conducted a review of the STGs used in the health centers, general hospitals,

Digital Features To view digital features for this article go to https://doi.org/10.6084/m9.figshare.12388859.

Electronic supplementary material The online version of this article (https://doi.org/10.1007/s40121020-00308-3) contains supplementary material, which is available to authorized users.

B. Arega $(\bowtie) \cdot A$. Agunie $\cdot$ A. Minda $\cdot$ A. Mersha . A. Sitotaw - G. Weldeyohhans - A. Teshome

Yekatit 12 Hospital Medical College, Addis Ababa, Ethiopia

e-mail: balewmlt@gmail.com

K. Adane

Meklle University, College of Health Science, Mekelle, Ethiopia

G. Mengistu

Debere Markos University, College of Health

Science, Debre Markos, Ethiopia and primary hospitals in Ethiopia and assessed the AMR recommendations in STGs for empirical antibacterial prescriptions for communityacquired pneumonia (CAP), urinary tract infection (UTI), tonsillopharyngitis, acute otitis media (AOM), and bacterial dysentery. Next, we performed an overview of AMR reviews published in Ethiopia. We used the MEDLINE/ PubMed, Embase, Cochrane Library, and Google Scholar databases to identify AMR reviews. The review characteristics were extracted. We also evaluated the quality of each included AMR review using a measurement tool to assess the systematic review scale (AMSTAR 2).

Results: A total of 6 STGs and 12 AMR reviews conducted in the country were included. The choice of empirical antibacterials for similar infectious syndromes (and editions) was comparable across the three levels of the health care system. None of the STGs evaluated included the local AMR recommendations for empirical antibacterial prescriptions for five common infectious syndromes. Of all the AMR reviews included, 75\% had low and below methodologic quality, and none had a high-quality score using the AMSTAR 2 tool.

Conclusion: Standard treatment guidelines did not consider local AMR recommendations for empirically prescribing antibacterials for common infectious syndromes. The AMR reviews published in the country produced poor methodologic quality evidence for clinical applications. This highlights the need to 
improve the methodologic quality to provide the best available evidence for clinical decisionmaking and curb the ongoing AMR in Ethiopia. Trial registration: Retrospectively registered (15/07/2020).

Keywords: Antimicrobial resistance; Ethiopia; Treatment guideline

\section{Key Summary Points}

\section{Why carry out this study?}

The national standard treatment guidelines (STGs) have become more appropriate because of using evidence on the antibacterial resistance (AMR) situation in Ethiopia.

Despite the growing number of STGs and AMR reviews in the country, a comprehensive evaluation of STGs' AMR recommendations and the quality of reviews on AMR was lacking.

This review describes the level of recommendations related to local AMR evidence in the STGs for empirical antibacterial prescriptions for five common infectious syndromes and the quality of the AMR reviews published since the national STGs were launched.

\section{What was learned from the study?}

None of the STGs evaluated included the local AMR recommendations for empirical antibiotic prescriptions for the five common infectious syndromes. More than $75 \%$ of the reviews on AMR in the county also had low and below quality, and none had a high-quality score according to the AMSTAR 2 tool.

\section{Reviews published on AMR produced} methodologically poor quality evidence for clinical applications. This highlights the need to improve the quality to provide the best available evidence for clinical decision-making and curb the ongoing AMR in the country.

\section{INTRODUCTION}

Antimicrobial resistance (AMR) has emerged as one of the principal public health problems of the twenty-first century, threatening the effective prevention and treatment of ever-increasing bacterial infections [1]. The problem is common mainly in low-income countries where microbiologic diagnostics are often nonexistent or weak and alternative antimicrobial agents are not commonly available [2].

The rapid spread of AMR has led to standardized treatment guidelines (STGs) being tailored according to the local resistance patterns of a given area [3]. The guidelines support clinical decision-making through a consensual process based on locally generated evidence on AMR and facilitate the best available choice of antibacterial agent, preventing relevant resistances [4]. This is effective when the guidelines are developed with the best available local research evidence from AMR reviews [systematic reviews with or without meta-analysis (SRs and MAs)] [5]. The reviews produce strong data for clinical applications when carried out using randomized clinical trials [6]. However, whether AMR reviews of observational studies generate important clinical evidence rather than improve the academic ranks of the reviewers is a significant concern [7].

Bacterial infections are the major cause of death in Ethiopia, and antibacterial resistance threatens the management of bacterial infection in the health care setting [8]. The first national AMR baseline survey (2009) revealed that most bacteria causing common infections in humans showed considerable resistance to commonly used first-line antibacterial agents [9]. The country's Medicine and Health Care Administration and Control Authority (FMHACA), currently called the EFDA (Ethiopia Food and Drug Administration), has established STGs for the three levels of the health care system (health centers, primary and general hospitals) since 2004 and has an updated third edition [10]. The guidelines aimed to ensure the effective and safe use of medicines, containing health care costs, and preventing antimicrobial resistance. However, a comprehensive 
evaluation of the local recommendations related to AMR for empirical antibacterial prescription for common infectious syndromes in the STGs was lacking. Furthermore, information on the quality of reviews on AMR, the potential sources of the recommendations to prepare STGs, in Ethiopia is not available. Therefore, the purpose of this study was twofold. First, it assessed the STGs' local antibacterial resistance recommendation level for empirically prescribing antibacterial agents for community-acquired pneumonia (CAP), urinary tract infections (UTIs), tonsillopharyngitis, bacterial dysentery, and acute otitis media (AOM). Second, it evaluated the methodologic quality of reviews on AMR published in Ethiopia using the AMSTAR 2 tool considering their use in clinical applications.

\section{METHODS}

This is a review of STGs used in the three health care levels (health centers, primary and general hospitals) and an overview of reviews on AMR related to bacteria isolated from human samples in Ethiopia.

\section{Search, Inclusion, and Data Extraction of STGs}

We retrieved all STGs published up to October 2019 from the FMHACA (currently EPDA) office and their official web site in Ethiopia [11]. We included the latest two editions for each health care level related to five common infectious syndromes [10, 12-16].

We extracted data about the types of infectious syndrome (CAP, UTIs, AOM tonsillopharyngitis, and bacterial dysentery), editions of STGs, and types of empirical antibacterial choices (first line, second line, or alternative). We also extracted the level of recommendations for empirical antibacterial prescriptions for the included clinical infection syndromes. The levels were classified into three categories as satisfactory, partially satisfactory, and unsatisfactory based on epidemiologic and resistance pattern data [5] (Table 1). Two authors (BA and GM) carried out the STG search and data
Table 1 Hierarchy of recommendations related to antimicrobial resistance in empirical therapy

Satisfactory: An alternative empirical antibiotic recommendation was supported by our countryspecific resistance patterns findings

Partial satisfactory: An alternative empirical antibiotic therapy recommendation was supported by inconsistent resistance patterns, which is not a country-specific finding

Unsatisfactory: The empirical antibiotic recommendation did not support any resistance patterns or was not justified by country-specific resistance patterns

extraction. We resolved the one-to-one disagreements that occurred during the search and data extraction by consulting a third person.

\section{Searching Reviews on AMR}

We included reviews on AMR (SRs and MAs) published in Ethiopia. We used the MEDLINE/ PubMed, Embase, Cochrane Library, and Google Scholar databases to search the original reviews. A hand search was also done for crossreference lists of the identified original reviews. A flow chart showing the selection of reviews was made following the Preferred Reporting Items for Systematic Reviews and Meta-Analyses (PRISMA) statement guideline [17]. An electronic search was performed using a combination of keywords for the MEDLINE/PubMed database using the Medical Science Heading (MeSH) terms: ["bacterial"(MeSHTerms) OR "bacteria"(AllFields)] OR ["microorganisms"(MeSHTerms) OR ["microorganisms" (All Fields) AND "antimicrobial resistance" (All Fields)] OR "antimicrobial resistance" [MeSH Terms] OR "drug-resistance" [All Fields] OR "drug-resistance" [MeSH Terms] AND ("metaanalysis and systemic review" [MeSH Terms] OR ["meta-analysis and systemic review" (All Fields) OR "systemic review" (All Fields)] OR "systemic review" (MeSHterm)] AND ["Ethiopia" (MeSH Terms) OR "Ethiopia" (All Fields)]. The reviews 
were limited to the human category. The data search was done between 1 September 2019 and 15 October 2019.

\section{AMR Review Inclusion and Data Extraction}

We included AMR reviews fulfilling the following criteria: (1) conducted in Ethiopia, (2) published and written in English, (3) took samples from humans (isolated from either diseased or healthy individuals), and (4) reported the antibacterial resistance. We excluded AMR reviews on tuberculosis, viruses, parasites, and fungi. We (BA, GM) conducted the data extraction using a standardized and pretested format in Microsoft Excel. The data extracted included: the the first author of the reviews, number of original studies in each review, study years in the original studies, review type (SR, MA), aims of the reviews, and the main/pooled/ resistance level of different antibacterial drugs mainly used for the treatment of the included infectious syndromes.

\section{Quality of AMR Reviews (SRs and MAs)}

Two reviewers (BA and GM) independently assessed the methodologic quality of each AMR review included in the overview using the AMSTAR 2 tool. This tool consists of 16 questions $(\mathrm{Q})$, each being answered with "yes," "partial yes," "no," or "no meta-analysis done." Of the 16 items or domains in the AMSTAR 2 framework, the creators of the tool specify 7 critical domains (items Q2, Q4, Q7, Q9, Q11, Q13, and A15) that can substantively impact the validity of a review. The other nine are noncritical domains (items Q1, Q2, Q5, Q6, Q8, Q10, 14Q, and Q16) (Supplement 1).

Following the evaluation of each of the seven critical domains as well as the nine noncritical domains, the overall result was rated as high quality, moderate quality, low quality, and critically low quality review (Supplement 2) [18]. A one-to-one disagreement that occurred during quality assessment between reviewers (BA and GM) was solved through by a third person.

\section{Compliance with Ethics Guidelines}

This article is based on previously conducted studies and does not contain any studies with human participants or animals performed by any of the authors.

\section{RESULTS}

\section{Standardize Treatment Guidelines and Recommendations}

From 2004-2019, nine STGs (three editions for each health care level) were developed by Ethiopia FMHACA. Of these, a total of six recent editions (the recent edition in 2014), two for each level of health care, were included for evaluation. The empirical antibacterial prescriptions in all STGs evaluated for the five clinical syndromes were described as first line and alternative. As presented in Table 2, the first line provided antibacterial drugs for similar clinical syndromes, and the STG editions are comparable for all three levels of the health care system. For instance, macrolides (clarithromycin or azithromycin) are the primary choice for CAP, a fluoroquinolone (ciprofloxacin or norfloxacin) for UTIs and bacterial dysentery, and penicillin (amoxicillin) for tonsillopharyngitis and AOM in the last STG editions for all the health care system levels.

Compliance with the desired criteria is shown in Table 1. Partially satisfactory recommendations related to antibacterial resistance and alternative antibacterial agents chosen for patients for recent antibacterial use were made for mild CAP in the latest editions of all health care system STGs. For all other clinical syndromes, the empirical antibacterial recommendation did not describe any resistance or found it was not justified by country-specific resistance patterns (unsatisfactory) in all STGs.

\section{Characteristics of AMR Reviews (SRs and MAs)}

Of the 146 AMR reviews identified, 21 were fully screened. Only 12 of them with a total of 312 


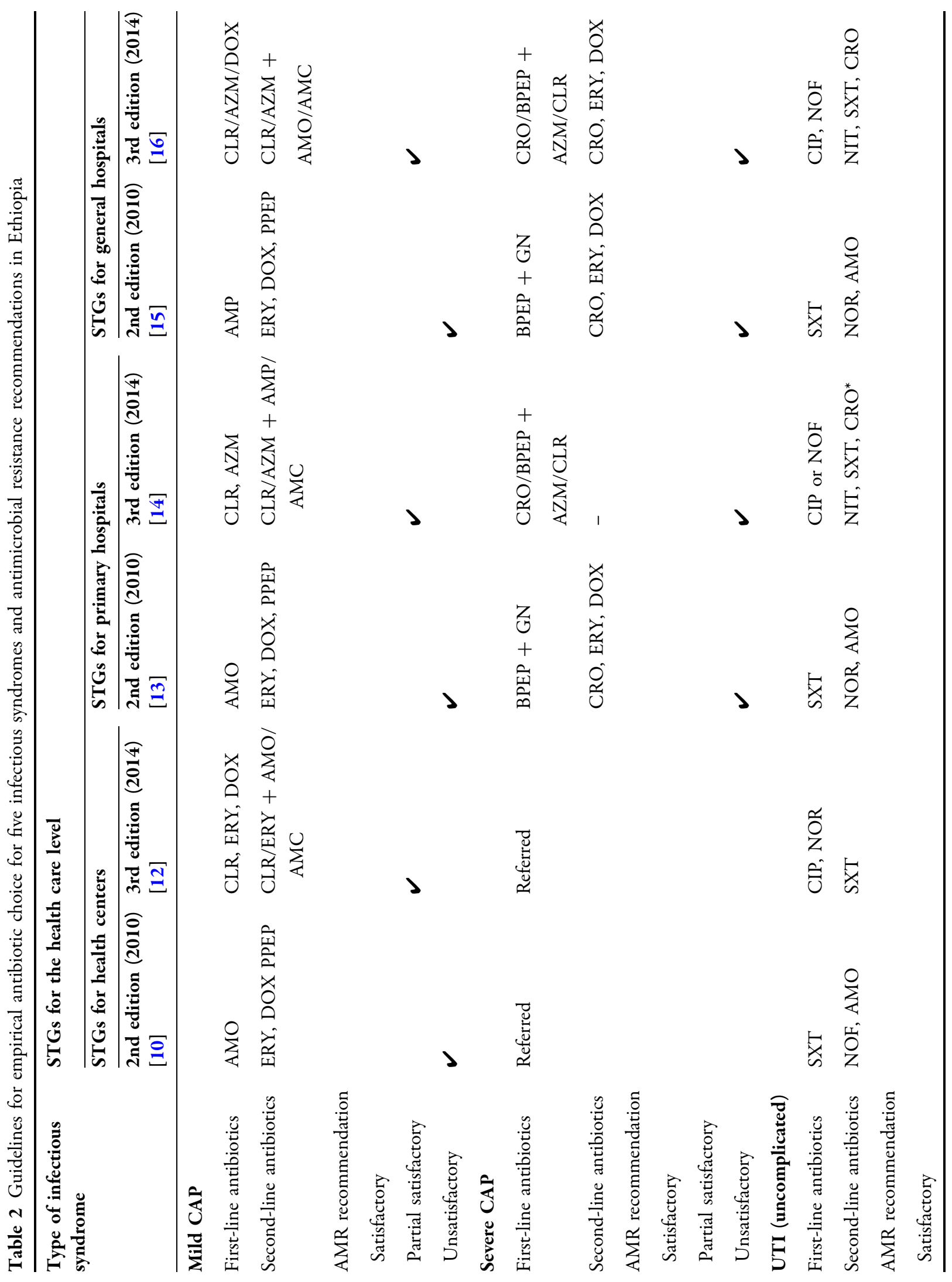




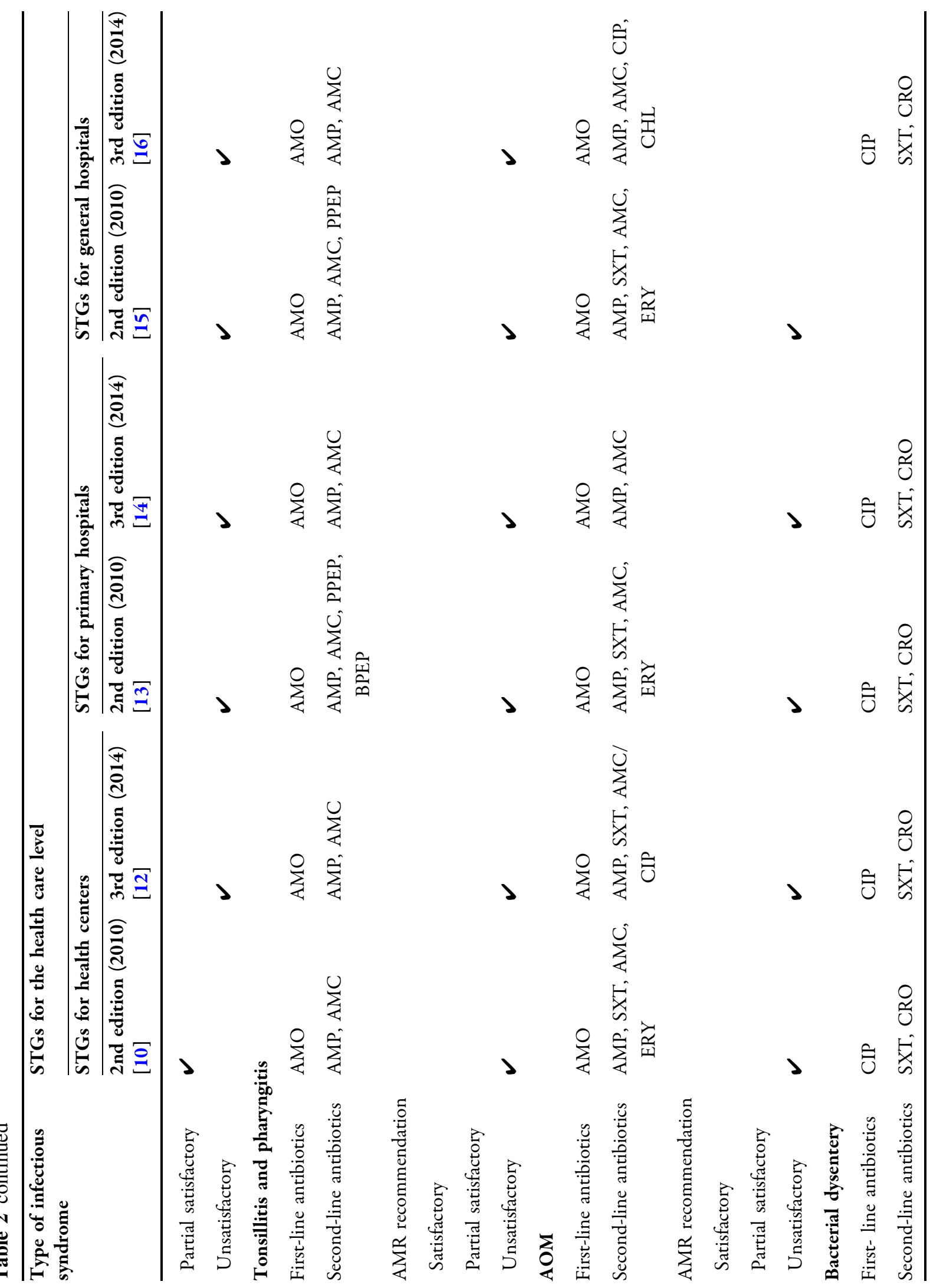




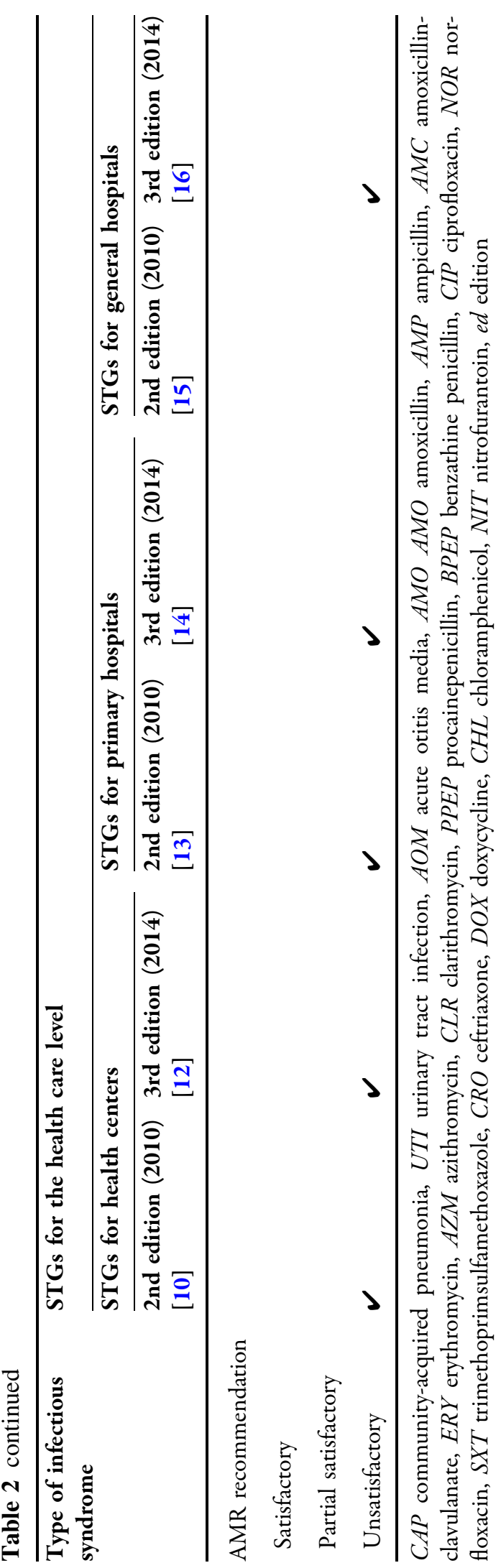

original studies fulfilled the inclusion criteria and were included in this overview (Fig. 1). The original studies were conducted between 1967 and 2018. Only two of the included AMR reviews [28-30] were SRs, and the remaining ten were both SRs and MAs [19-27]. Four AMR reviews reviewed the AMR profile of gram-positive bacteria (GPB) $[20,21,26,28]$, four reviewed the AMR of gram-negative bacteria (GNB) $[19,24,25,30]$, and four reviewed the AMR of both GPB and GNB [23, 24, 27, 29].

The pooled resistance level of different bacteria species for commonly used antibacterial drugs is shown in Table 3. The resistance level of ampicillin (AMP) was 64-98.1\% [19-24, 26], amoxicillin (AMO) 40-97\% [20-22, 24-26], methicillin-resistant $S$. aureus (MRSA) 10.94$49 \%$ [20, 21, 23-26], ceftriaxone (CRO) $9.3-45 \%$ [22-27], erythromycin (ERY) 30-97.1\% [20-22, 24-26], ciprofloxacin (CIP) 3.6-35.1\% [19-24], and trimethoprim-sulfamethoxazole (SXT) 35-68\% [19-21, 24-26].

\section{Quality of AMR Reviews (SRs and MAs) Using AMSTAR 2 Items}

Table 4 presents the results of each AMR review according to the AMSTAR 2 questions and their quality scores. All of the AMR reviews implemented components of PICO for observational studies (Q1) and the absence of a statement of conflict of interest (Q16). In $>50 \%$ of the reviews, the original studies provided adequate detail (Q8), provided a satisfactory explanation for any heterogeneity observed (Q14), used appropriate methods for a statistical combination of results (Q11), assessed the potential impact of risk of bias on the overall results (Q12), performed data extraction in duplicate (Q6), explained their selection of study design in the included studies (Q3), used a comprehensive literature search strategy(Q4), and performed study selection in duplicate (Q5). None of the reviews reported on the sources of funding for the studies included in their review (supplement 3). 


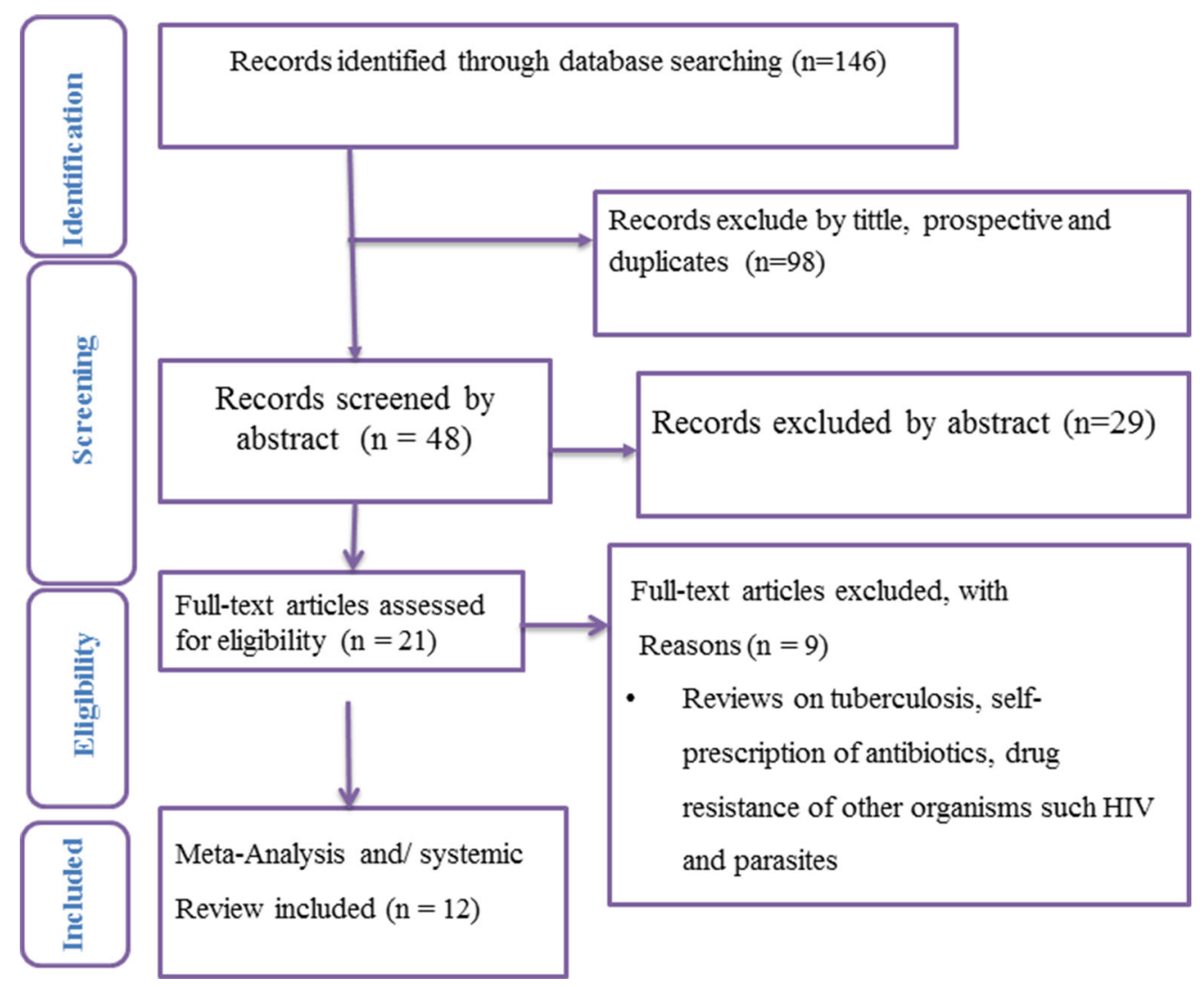

Fig. 1 PRISMA flow chart of review selection

\section{Overall Quality Score of AMR Reviews Using the AMSTAR 2 Tool}

Overall, three-quarters of the included AMR reviews had low quality and less overall, onefourth had moderate quality, and none had a high-quality score using the AMSTAR 2 score (Supplement 4).

\section{DISCUSSION}

In the era of the global burden of AMR, the treatment for a growing number of infectious diseases mandates recommendations about AMR in the clinical treatment guidelines to help reduce resistance [31]. To the best of our knowledge, this is the first study to review the STGs and evaluate the quality of AMR reviews in Ethiopia, trying to link the evidence to clinical application. The overview found two important gaps: (1) The STGs did not routinely consider local resistance patterns for empirical antibacterial prescription for the five highly prevalent infectious syndromes. (2) The AMR reviews published in the country produced evidence with poor methodologic quality for clinical application.

In this study, the majority of clinical treatment guidelines (health center, primary and general hospital) have unsatisfactory AMR recommendations for empirical antibacterial therapy; these findings are similar to others in which current guidelines elsewhere do not have resistance recommendations. In this regard, a recent survey revealed that only 16 of 135 (6.4\%) guidelines discussed empirical antibiotic treatment considering specific microbiology resistance [5]. In contrast, the WHO clinical treatment guideline development protocols strongly recommend consideration of the local resistance pattern for empirical therapy, as resistance often differs across geographical regions or even within different health settings 


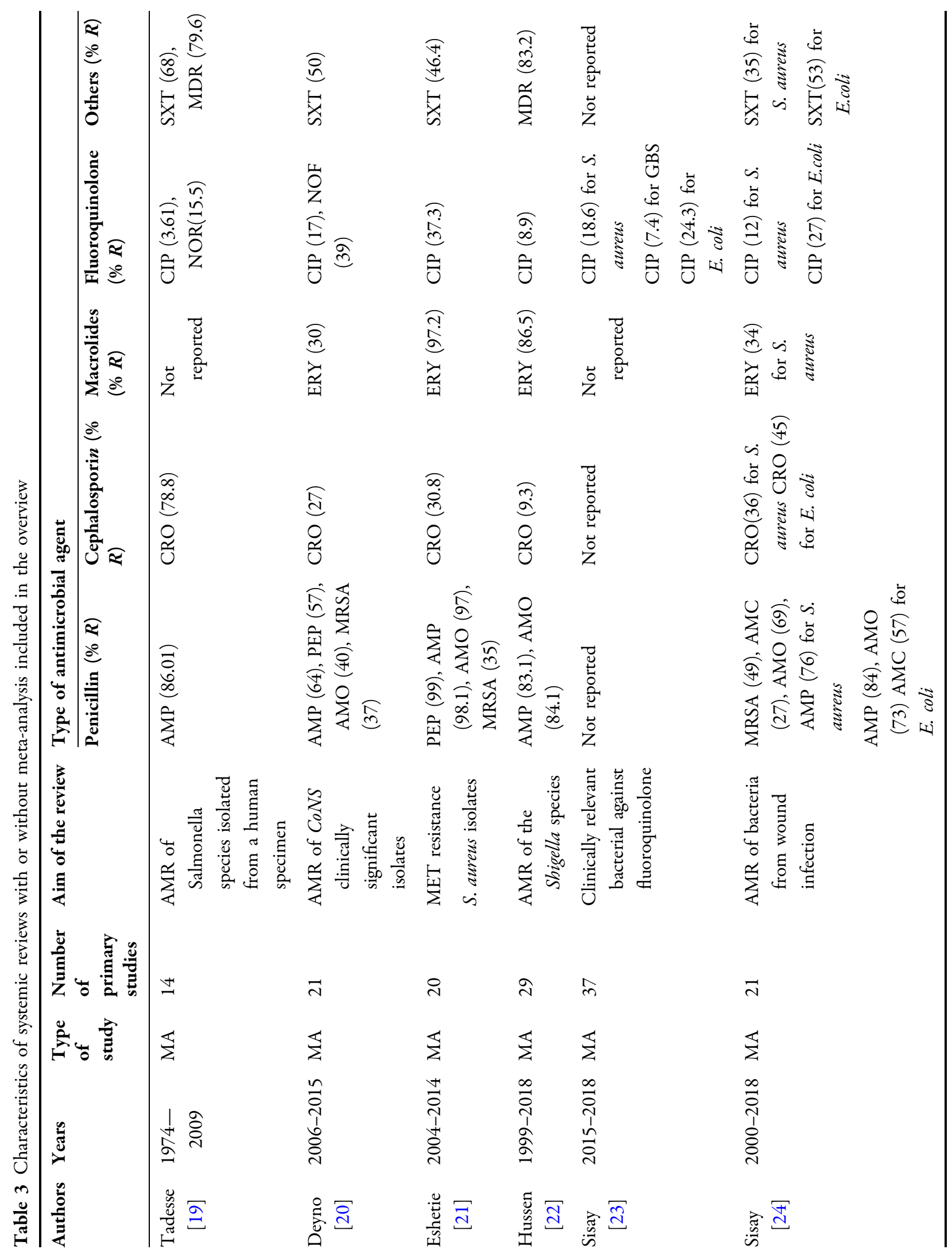




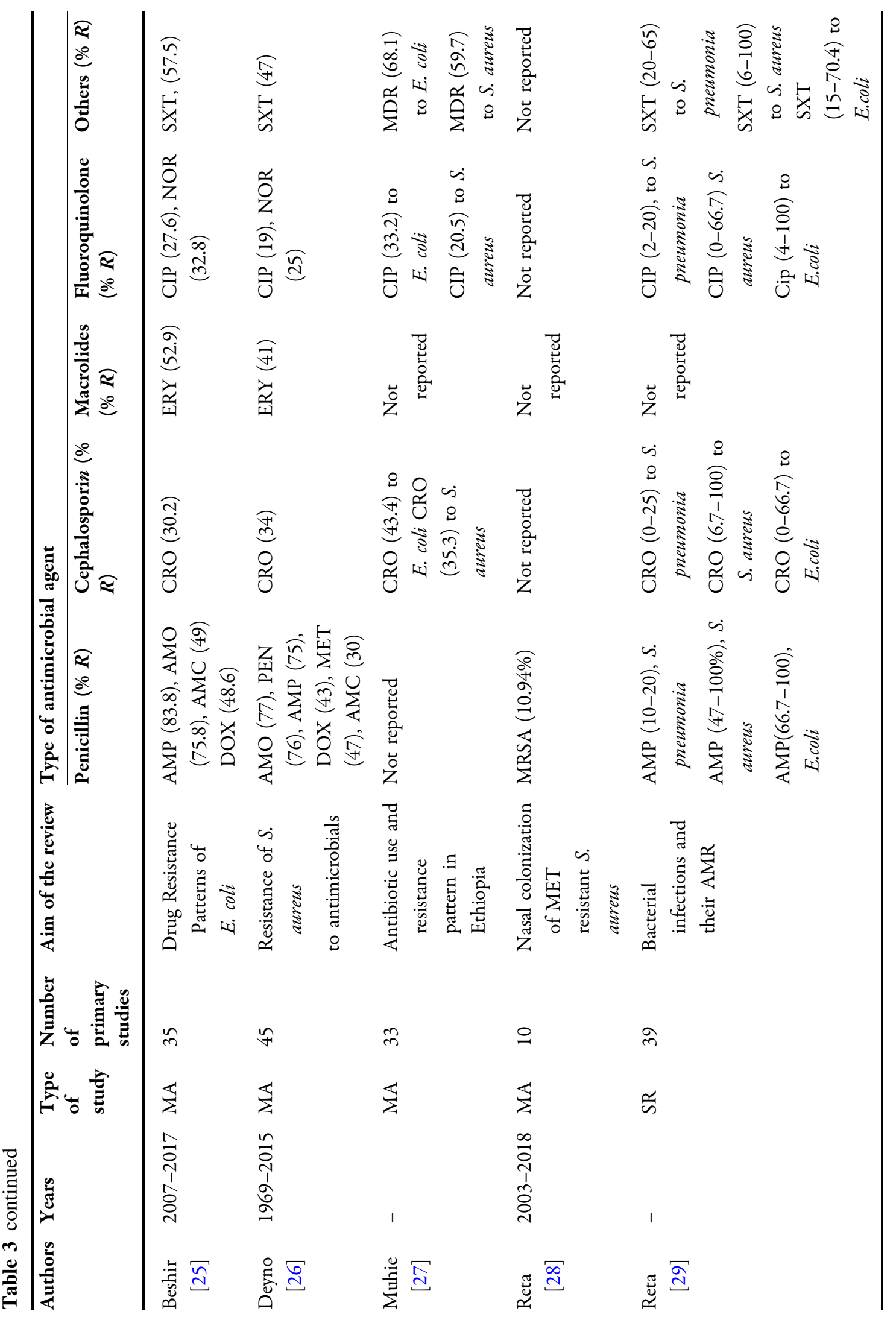



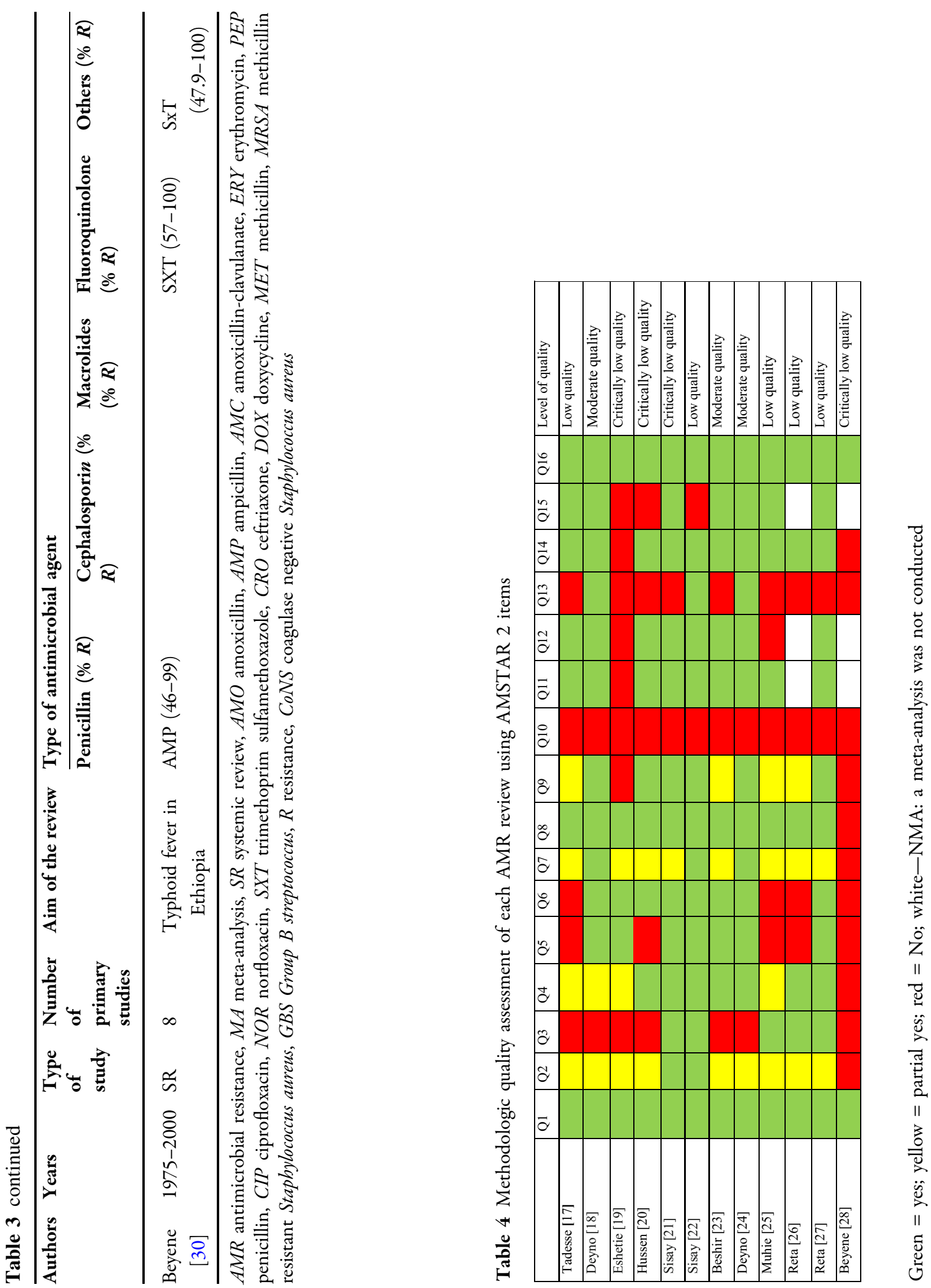
in the same country [32]. Recommendations related to AMR in the STGs are especially essential in low-income countries including Ethiopia where the antibiogram profile of each isolated bacteria to prevent the spread and development of resistance in the country has not been established for several reasons. If resistance is not described in a guideline, it is unlikely to consider modification of the antibacterial agent prescribed. Therefore, the findings of these reviews imply that significant changes in the components of STGs are needed to improve the way resistance data recommendations are made.

In Ethiopia, patients are referred to the next higher health care system, from a health center to primary hospital to general hospital, when the cases are complicated or patients do not respond to the available empirical antibacterial therapy [32]. Therefore, patients in the higher health care level could have a history of taking multiple antibiotics, one of the major risk factors for AMR [33]. Antibiotic prescriptions in the higher care levels logically should either be based on specific microbiologic data, which is not routinely practiced in our settings [34], or the guideline should have a strong recommendation about AMR. Interestingly, however, our investigation into the guidelines revealed that the types of antibacterial agent recommended for a similar syndrome (in similar editions) across the levels of health care systems were almost equivalent. This indicates that guidelines could be potential sources of antimicrobial resistance as they permit individuals to take multiple similar antibiotics at the different health care levels.

The quality of clinical treatment guidelines, on the other hand, depends on the quality of evidence used for the development of the guidelines or recommendations, including specific cases within the content of the guidelines [35]. Therefore, a well-designed systematic review or meta-analysis should be performed to provide healthcare providers with the best available evidence. In Ethiopia, even though the rate of publication of reviews has risen in the last decade, their methodologic quality has been doubtful. This could be related to conducting reviews without registering a study protocol, publishing in low impact journals, absence of funding, introducing bias risks in the studies, or using weak study designs [36]. In this overview, three-quarters of AMR reviews had low quality or less, and none had high methodologic quality. Consistent with our finding, an overview in the country, not limited to AMR, found that three-quarters of the reviews had poor methodologic quality [37]. Similarly, another overview assessing reviews of antibiotic use among livestock showed that $85 \%$ had critically low quality [38]. The major weaknesses of reviews included in our overview were related to the assessment of sources of funding for the original studies, discussing the risk of bias in the individual studies, study selection in duplicate, data extraction in duplicate, and adequate investigation of publication bias. The aforementioned overviews also reported that none of the reviews reported sources of funding for the primary studies [37, 38]. Therefore, we might conclude that the pooled estimates reported in the reviews and in our country were susceptible to methodologic flaws, which seriously weaken the confidence in using the finding for clinical decisions. Good-quality research effects positive changes in patient care and decreases the ongoing threat of antimicrobial resistance in the country [39]. Therefore, Ethiopian researchers should focus on using strong study designs such as a randomized clinical trials, cohort-based studies, and large multidisciplinary registered reviews rather than mainly observational studies [39]. Constraints on Ethiopia's researchers include the limited national budget and small number of open grant opportunities, which could be minimized by involvement and winning medium and large international and regional grants [36].

While the quality of the SRs and MAs was heterogeneous with most having a low quality score or less, the pooled resistance patterns of the common antimicrobial agents used for the treatment of the five infectious syndromes in our health settings showed a high resistance level. In this case, the resistance of AMO, AMP, and SXT ranged from 35 to $98.1 \%$. Similarly, high levels of AMR (50-100\%) to commonly used antibiotics (including AMP and SXT) were also reported in a systemic review in east Africa 
[40]. In addition, macrolides generally and erythromycin/clarithromycin in particular are the recommended first-line empirical antibiotics prescribed for community-acquired pneumonia in Ethiopian STGs. Six of the reviewed SRs and MAs (three moderate $[20,25,26]$ and three critically low quality $[21,22,24])$ revealed significantly high resistance of erythromycin (30-97.6\%). According to the FMHACA national baseline, AMR surveillance reported that the resistance of Streptococcus pneumonia, the most common cause of CAP, to erythromycin increased from $0 \%$ in 1996 to $19.2 \%$ in 2000 [9]. In Ethiopia, where clinicians are not supported by basic bacteriology services to ensure that treatment is tailored to the specific condition, information deduced from such pooled data is crucial in principle. However, the poor quality of the majority of reviews hinders the opportunity to develop and use evidencebased guidelines for recommendations related to AMR for empirical therapy, which would help control AMR, improve patient outcomes, and allow early recognition of treatment failure.

The study had certain limitations: Although the AMSTAR 2 tool is designed to critically appraise systematic reviews, both randomized and non-randomized systemic reviews, of healthcare interventions, there are some weaknesses of the criteria. First, two of the items in the AMSTAR 2 tool (items 12 and 13) suggest that the authors should only include randomized controlled trials with a low risk of bias in a meta-analysis or need to discuss the potential impact of including studies with a higher risk of bias. The tool does not include an elaboration of the appropriate features that such a discussion should include so objective evaluation of these criteria is difficult. Second, some items on the AMSTAR 2 checklist are more relevant to the completeness of reporting as opposed to methodologic quality. Specifically, items 1, 8, 10 , and 16 concern the reporting of the review research question, details of the studies included in the review, funding sources for the included studies, and sources of funding for the review, respectively. Although being clear and comprehensive is essential, research quality and research reporting are separate issues. Third, the eighth AMSTAR 2 checklist is more relevant to the completeness of reporting as opposed to methodologic quality. Third, it is also important to acknowledge that it has yet to be validated [18]. Lastly, given the limited number of reviews, including only 12 SRs and MAs, a topicbased in-depth analysis was not carried out, and the results should be interpreted with caution.

\section{CONCLUSION}

Our study revealed that standard clinical treatment guidelines did not routinely consider the local antimicrobial resistance of the five infectious syndromes. The antimicrobial resistance reviews published in Ethiopia had poor methodologic quality scores using the AMSTAR 2 tool. Therefore, to improve clinical decisionmaking, researchers in Ethiopia should focus on improving the quality of AMR reviews rather than continuing to publish in quantity. Future research is needed to investigate the strategies to improve the methodologic and reporting quality of systematic reviews or meta-analyses on AMR in Ethiopia.

\section{ACKNOWLEDGEMENTS}

We thank the Food, Medicine, and Health Care Administration and Control Authority of Ethiopia for providing and allowing the use of standardized treatment guidelines for this study.

Funding. No funding or sponsorship was received for this study or publication of this article.

Authorship. All named authors meet the International Committee of Medical Journal Editors (ICMJE) criteria for authorship for this article, take responsibility for the integrity of the work as a whole, and have given their approval for this version to be published.

Disclosures. Balew Arega, Asnake Agunie, Abraham Minda, Amdemeskel Mersha, Alazar Sitotaw, Getachew Weldeyohhans, Ayele Teshome, Kelemework Adane, and Getachew 
Mengistu declare that they have no conflict of interest.

Compliance with Ethics Guidelines. This article is based on previously conducted studies and does not contain any studies with human participants or animals performed by any of the authors.

Data Availability. All data generated or analyzed during this study are included in this published article/assupplementary information files.

Open Access. This article is licensed under a Creative Commons Attribution-NonCommercial 4.0 International License, which permits any non-commercial use, sharing, adaptation, distribution and reproduction in any medium or format, as long as you give appropriate credit to the original author(s) and the source, provide a link to the Creative Commons licence, and indicate if changes were made. The images or other third party material in this article are included in the article's Creative Commons licence, unless indicated otherwise in a credit line to the material. If material is not included in the article's Creative Commons licence and your intended use is not permitted by statutory regulation or exceeds the permitted use, you will need to obtain permission directly from the copyright holder. To view a copy of this licence, visit http://creativecommons.org/licenses/bync/4.0/.

\section{REFERENCES}

1. Cosgrove SE. The relationship between antimicrobial resistance and patient outcomes: mortality, length of hospital stay, and health care costs. Clin Infect Dis. 2006;42(2):S82.

2. Ayukekbong JA, Ntemgwa M, Atabe AN. The threat of antimicrobial resistance in developing countries: causes and control strategies. Antimicrob Resist Infect Control. 2017;6:47.

3. Aseffa A, Abate E, Seyoum B, et al. Investigations on antimicrobial resistance: contributions of a clinical research network. Ethiop Med J. 2019;1:3-7.
4. Yusuf E, Ong DS, Martin-Quiros A, et al. A large survey among European trainees in clinical microbiology and infectious disease on training systems and training adequacy: identifying the gaps and suggesting improvements. Eur J Clin Microbiol Infect Dis. 2017;36:233-42.

5. Elias C, Moja L, Mertz D, et al. Guideline recommendations and antimicrobial resistance: the need for a change. BMJ Open. 2017;7:e016264.

6. WHO. WHO handbook for guideline development. 2012.

7. Prorok JC, Iserman EC, Wilczynski NL, et al. The quality, breadth, and timeliness of content updating vary substantially for 10 online medical texts: an analytic survey. J Clin Epidemiol. 2012;65: 1289-95.

8. Hazim C, Abubeker Ibrahim R, Westercamp M, et al. Establishment of a sentinel laboratory-based antimicrobial resistance surveillance network in Ethiopia. Health Secur. 2018;16(S1):S30-6. https:// doi.org/10.1089/hs.2018.0052.

9. Ibrahim RA, Amete M. Antimicrobial resistance surveillance in Ethiopia: implementation experiences and lessons learned and Wubshet M. KefichoAfr. J Lab Med. 2019;06;8(1):1109.

10. Food, Medicine, and Health Care Administration and Control Authority. Standard Treatment Guideline for health centers. Third Edition, 2010

11. Ethiopian Food and Drug Authority. Available: http://www.fmhaca.gov.et/covid19 publications. Accessed 15 Oct 2019.

12. Food, Medicine, and Health Care Administration and Control Authority. Standard treatment guideline for health cenetrs. Addis Ababa: Food, Medicine, and Health Care Administration and Control Authority; 2014.

13. Food, Medicine, and Health Care Administration and Control Authority. Standard treatment guidelines for primary hospitals. Addis Ababa: Food, Medicine, and Health Care Administration and Control Authority; 2010.

14. Food, Medicine, and Health Care Administration and Control Authority. Standard treatment guidelines for primary hospitals. Addis Ababa: Food, Medicine, and Health Care Administration and Control Authority; 2014.

15. Food, Medicine, and Health care Administration and control authority. Standard treatment guidelines for general hospitals. Third Edition, 2010. 
16. Food, Medicine, and Health care Administration and control authority. Standard treatment guidelines for general hospitals. Third Edition, 2014.

17. Moher D, Liberati A, Tetzlaff J, Altman DG, the PRISMA Group. Preferred reporting items for systematic reviews and meta-analyses: the Prisma statement. PLoS Med. 2009;6(6):e1000097.

18. Shea BJ, Reeves BC, Wells G, et al. AMSTAR 2: a critical appraisal tool for systematic reviews that include randomized or non-randomized studies of healthcare interventions, or both. BMJ. 2017;21: 358:j4008.

19. Tadesse G. A meta-analysis of the proportion of antimicrobial resistant human Salmonella isolates in Ethiopia. BMC Pharmacol Toxicol. 2014;15:51.

20. Deyno S, SintayehuFekadu S, Seyfe S. Prevalence and antimicrobial resistance of coagulase-negative staphylococci clinical isolates from Ethiopia: a meta-analysis. BMC Microbiol. 2018;18:43.

21. Eshetie S, Tarekegn F, Moges F. Methicillin-resistant Staphylococcus aureus in Ethiopia: a meta-analysis. BMC Infect Dis. 2016;16:689.

22. Hussen S, Mulatu G, Kassa ZY. Prevalence of Shigella species and its drug resistance pattern in Ethiopia: a systematic review and meta-analysis. Ann Clin Microbiol Antimicrob. 2019;18:22.

23. Sisay M, Weldegebrea F, Tesfa T. Resistance profile of clinically relevant bacterial isolates against fluoroquinolone in Ethiopia: a systematic review and meta-analysis. BMC Pharmacol Toxicol. 2018;19: 86.

24. Sisay M, Worku T, Edessa D. Microbial epidemiology and antimicrobial resistance patterns of wound infection in Ethiopia: a meta-analysis of laboratorybased cross-sectional studies. BMC Pharmacol Toxicol. 2019;20:35.

25. Tuem KB, Gebre AK, Atey TM. Drug resistance patterns of Escherichia coli in Ethiopia: a meta-analysis. BioMed Research International 2018. https://doi. org/10.1155/2018/4536905.

26. Deyno S, Fekadu S, Astatkie A. Resistance of Staphylococcus aureus to antimicrobial agents in Ethiopia: a meta-analysis. Antimicrob Resist Infect Control. 2017;6:85.

27. Muhie OA. Antibiotic use and resistance pattern in Ethiopia: systematic review and meta-analysis. Int J Microbiol. 2019. https://doi.org/10.1155/2019/ 2489063.
28. Reta A, Mengist A, Tesfahun A. Nasal colonization of methicillin-resistant Staphylococcus aureus in Ethiopia: a systematic review and meta-analysis. Ann Clin Microbiol Antimicrob. 2019;18:25.

29. Reta A ,Kifilie AB, Mengist A. Bacterial infections and their antibiotic resistance pattern in Ethiopia: a systematic review Hindawi. Adv Prevent Med. 2019. https://doi.org/10.1155/2019/4380309.

30. Beyene G, Asrat D, Mengistu Y. Typhoid fever in Ethiopia. J Infect Dev Ctries. 2008;2(6):448-53.

31. WHO. Report on surveillance of antibiotic consumption. 2016-2018.

32. Federal democratic republic of Ethiopia ministry of health. Guideline for implementation of a patient referral system. May 2010.

33. WHO. Implementation of the global action plan on antimicrobial resistance. WHO gap AMR;2017.

34. Gebretekle GB, Haile Mariam D, Abebe W, et al. Opportunities and barriers to implementing antibiotic stewardship in low and middle-income countries: lessons from a mixed-methods study in a tertiary care hospital in Ethiopia. PLoS ONE. 2018;13(12):e0208447.

35. American a cademy of pediatrics.classifying recommendations for clinical practice guidelines. Pediatrics 2004;114(3):874-6.

36. Tesfa B. Challenges of Ethiopian researchers and potential mitigation with Ethiopian science, technology, and innovation policy 2015; Corpus ID: 14798318:1-17.

37. Habtewold TD, Alemu SM, Mohammed SH, et al. Reviews and meta-analyses in Ethiopia had poor methodological quality: an overview of evidence 1970 to 2018. J Clin Epidemiol. 2019;109:90-8.

38. Vriezen R, Sargeant JM, Vriezen E, Winder CB, O'Connor AM. Quality assessment of systematic reviews and meta-analyses that examine preventive antibiotic uses and management practices designed to prevent disease in livestock. Anim Health Res Rev. 2019;20:305-18.

39. Gaym A. Health research in Ethiopia-past, present, and suggestions on the way forward. Ethiop Med J. 2008;46(3):287-308.

40. Ampaire L, Muhindo A, Orikiriza P, et al. A review of antimicrobial resistance in East Africa. Afr J Lab Med. 2016;5(1):1-6. 